\title{
Un balance de la modernización autonómica y las tensiones intergubernamentales
}

\author{
María Ángeles ABELLÁN LÓPEZ \\ Universidad Miguel Hernández \\ ma.abellan@umh.es
}

Recibido: 09/04/2015

Aceptado: 15/09/2015

\begin{abstract}
Resumen
En este artículo se propone una visión panorámica de la modernización de las administraciones autonómicas desde su creación hasta la actualidad, en el marco de las relaciones intergubernamentales, sin ánimo de exhaustividad. Diferenciamos, grosso modo, cuatro ciclos modernizadores: el primero se identifica con la búsqueda de legitimidad y supervivencia de las administraciones autonómicas. El segundo se inspira en la Nueva Gestión Pública (NGP) y la irrupción de un paradigma postburocrático que posibilita el desarrollo de nuevas metodologías. El tercer ciclo modernizador se caracteriza por la primacía de la e-administración y la calidad de los servicios públicos. Finalmente, el último ciclo plantea tendencias antagónicas, según su ubiquen antes o después del estallido de la actual crisis económica.
\end{abstract}

Palabras clave: Comunidades Autónomas, modernización, reformas estructurales, relaciones intergubernamentales, Administración General del Estado.

\section{A balance of economic modernization and intergovernmental tensions}

\begin{abstract}
This article contains an overview of the modernization of the regional administrations since its inception until present. We distinguish roughly four modernizing cycles: the first is mainly identified with the search for legitimacy and survival of the regional administrations. The second is inspired by the New Public Management (NPM) and the emergence of a post-burocratic paradigm that enables the development of new methodologies. The third is characterized by the primacy of $e$-administration and quality policies applied to public services. Finally, the last cycle poses antagonistic tendencies, according to the outbreak of the current economic crisis.
\end{abstract}

Key words: autonomous communities, modernization, structural reforms, intergovernmental relations, General State Administration.

\section{Planteamiento general}

Se han cumplido treinta y cinco años desde que el proceso de descentralización autonómico iniciara su andadura y una mirada retrospectiva, sobre la transformación polí- 
tica administrativa de España, nos indica que el proceso está consolidado pero con grandes retos pendientes que afrontar. Todos los procesos de cambio administrativo siempre serán algo inacabado pues no implican normalmente cambios totales o radicales, sino que suponen la suma de realidades diferentes y, a veces, contradictorias (Canales Aliende, 1996:139). La modernización administrativa abarca un proceso amplio y complejo de adaptación y respuesta al entorno por parte de las administraciones públicas vinculado a cambios sociales y políticos, mientras que el término reforma se refiere a un cambio en el diseño formal del sector público, acompañado de un cambio normativo, presupuestario y procedimental (Canales Aliende, 2002:76). O dicho de otro modo, la reforma responde a la lógica del plan y la modernización a la de la estrategia (Prats, 2010:48).

La modernización de las administraciones públicas tiene como gran objetivo el cumplimiento de los fines que tiene encomendados el aparato estatal para servir a la sociedad a la que está vinculado, prestar mejores servicios públicos a los ciudadanos y contribuir a la cohesión social. El fundamento último es la convicción de que no puede haber una buena sociedad sostenible sin una buena administración (Prats, 2010:51).

Si bien como se ha señalado, la modernización administrativa se incardina dentro de un proceso global mucho más amplio, que en el caso español ha de enmarcarse dentro del proceso de descentralización política experimentado por el sistema político administrativo español unido a la introducción del enfoque de la Nueva Gestión Pública (NGP) (Morata, 1992, 2001; OCDE, 1997; Bañón y Carrillo, 1997; Olías de Lima, 2001; Canales Aliende, 1996, 2002; Barzelay, 2002; Ramió y Salvador, 2004; Aguilar Villanueva, 2006; Carro Fernández-Valmayor, 2011).

Sin embargo, existen nuevos desafíos de viejos problemas no solucionados históricamente y que la crisis económica, social e institucional desde 2008 ha recrudecido con virulencia. Los grandes retos del Estado Autonómico son numerosos y de gran calado político como: a) las complejas relaciones intergubernamentales, b) el proceso soberanista catalán, c) la búsqueda del diseño óptimo y justo de la financiación autonómica para garantizar la autonomía y la igualdad, d) el establecimiento de dispositivos potentes y eficaces de coordinación horizontal, e) el refuerzo de la corresponsabilidad política fiscal y la rendición de cuentas de los gobiernos regionales y, f) la construcción de una cultura institucional cooperativa. En un contexto de crisis económica y de primacía de la reducción del déficit fiscal y de la deuda pública, para el que se ha puesto en marcha una agenda plena de reformas, el apoyo de las comunidades autónomas resulta imprescindible para su éxito. La OCDE (2014) sostiene que sin el concurso autonómico, dentro de un marco de gobernanza pública, las reformas puestas en marcha por el Estado están abocadas al fracaso. Un enfoque integral de los problemas de gobierno requiere un diálogo permanente entre los actores implicados y las esferas multinivel de gobierno. En este marco nos podemos preguntar: ¿Pueden ser modernizadas las administraciones públicas españolas con una escasa participación de las comunidades autónomas en ese proceso? ¿Cabe desarrollar e implantar medidas e instrumentos modernizadores novedosos con ausencia de la cooperación intergubernamental horizontal? 
Desde nuestro punto de vista, difícilmente pueden articularse con éxito medidas modernizadoras de las administraciones públicas sin el concurso activo de las comunidades autónomas, puesto que la esencia de las actuaciones cooperativas debe ir más allá de una mera instrumentalidad jurídica y fundamentarse en la voluntad real de colaboración.

El objetivo que se plantea a lo largo de las siguientes líneas será describir, a grandes rasgos, el proceso de modernización administrativo durante las tres décadas transcurridas, enfatizando determinados aspectos de las relaciones intergubernamentales como parte de un diseño institucional aún por perfeccionar y que supone un importante reto para una futura modernización de la gestión pública.

\section{La construcción del diseño autonómico}

El sistema político español inició a finales de los años setenta del pasado siglo una profunda transformación que, no solo supuso un referente de cambio político administrativo de enorme magnitud, sino una metamorfosis de su sociedad y de su cultura cívica-política. Sin embargo, estas mutaciones tan significativas no se reflejaron en innovaciones destacadas en las administraciones públicas regionales que tomaron como modelo el de la AGE. Por consiguiente, el proceso de creación institucional apareció influido por la Administración del Estado en cuanto único referente válido (Rodriguez-Arana, 1996). La Constitución española (CE) de 1978, en su título VIII, proyectó un Estado descentralizado y poliédrico, que rompía con el modelo centralista del régimen franquista e incorporaba novedades político institucionales de gran calado. El Estado descentralizado se ha desarrollado durante las tres últimas décadas en un triple nivel institucional: Estado Central, sistema autonómico y régimen local (Canales y Menéndez, 2013:15). Esta situación ha supuesto la superposición de redes de centro de poder político administrativo, lo que ha configurado una más compleja distribución territorial del poder. La Carta Magna no define el diseño autonómico ni establece las competencias y la organización de sus instituciones de autogobierno pero lo que sí hace es plasmar los principios y procedimientos que han de regir la constitución e institucionalización de las comunidades autónomas. La CE no establece un sistema federal sino un modelo de geometría variable (Morata, 2001:124). Las vías que dan acceso a la formación de los entes autonómicos son el artículo 151, el artículo 143 y el 144 (mediante autorización de las Cortes), que se corresponden con los tres procedimientos que permiten el acceso al autogobierno autonómico: el procedimiento común, especial y el excepcional (Baena del Alcázar, 1990: 269). En este escenario de reparto competencial nuestro país inaugura la gobernanza pública multinivel que desde su comienzo se perciben marcadas tensiones interterritoriales. Los principales núcleos de problemas sobre los que giró la construcción del Estado Autonómico y que no han perdido su vigencia son las relaciones intergubernamentales, el déficit de cultura institucional cooperativa y la financiación autonómica. 


\subsection{Las tensiones intergubernamentales y el déficit cooperativo del Estado}

Las relaciones intergubernamentales ${ }^{1}$ han estado presididas tradicionalmente por el recelo y la desconfianza. La complejidad del sistema competencial, el deseo de afirmación de las nuevas élites políticas y las inercias centralistas condujeron a una rápida expansión de los conflictos entre ambos niveles gubernamentales, teniendo en cuenta que en el desarrollo de la planta autonómica, la acción normativa estatal básica ha sido un punto de conflicto y debate constante con las CC.AA. Algunos litigios tienen largo recorrido como el déficit de cooperación, la heterogeneidad de las competencias autonómicas y el rechazo de Cataluña ${ }^{2}$ y el País Vasco a la fórmula «café para todos».

El modelo de relaciones intergubernamentales se ha caracterizado por una escasa colaboración y coordinación entre todos los actores implicados. En todo caso, esta colaboración se ha articulado a través de dispositivos formales de cooperación, con un claro predominio de las relaciones intergubernamentales verticales sobre las horizontales y de la bilateralidad sobre la multilateralidad (Jiménez Asencio, 2010). La desconfianza entre Estado y CC.AA. es de tipo político y se parte de la visión errónea de que cualquier tipo de cooperación de las regiones entre sí obedece necesariamente a tendencias centrífugas (Santolaya Machetti, 1984).

Otro de los frentes que ha condicionado el desarrollo institucional de las CCAA ha procedido de las resistencias de la AGE a la realidad descentralizadora. La falta de una cultura institucional de colaboración y de cooperación entre las partes implicadas transmite rigideces y bloqueos que se sustancian en un clima de conflictividad intergubernamental ${ }^{3}$.

${ }^{1}$ Por relaciones intergubernamentales (RIG) seguimos la propuesta de definición de Anderson (citado por Agranoff, 1996:127) como «el contingente de actividades o interacciones que tienen lugar entre unidades de gobierno de todo tipo y nivel territorial de actuación». Cada una de estas interacciones o redes se rigen por patrones diversos como: intercambio de recursos, el dominio organizativo, los conflictos parciales las fórmulas de autoridad y dependencias de poder (Canales Aliende y Pérez Guerrero, 2002:92).

La RIG se caracterizan por los siguientes rasgos: 1) incluyen una amplia gama de relaciones multinivel entre las diferentes unidades; 2) destaca la importancia del elemento humano como promotores de las conductas interactivas que vinculan a los empleados públicos en ámbitos multinivel; 3) los actores implicados -empleados públicos- intercambian información y buscan la viabilidad y prosperidad de los asuntos en liza, y para tal fin, utilizan mecanismo formales, informales, prácticos y orientados a los fines perseguidos; 4) Las RIG poseen una dimensión política entendida como el conjunto de intenciones y acciones o inacciones de los empleados públicos y las consecuencias de sus acciones (Wright citado por Agranoff, 1996: 127-128). El concepto de RIG trasciende el enfoque meramente legalista y formal.

2 El proceso soberanista catalán puede considerarse entre los conflictos más serios de nuestro sistema autonómico enmarcado en un contexto económico con restricciones severas de gasto público, una alta deuda pública y centrado en el ejercicio del denominado «derecho a decidir» como fórmula de avance hacia la independencia. La cuestión catalana, tras la convocatoria de una consulta ciudadana por la Generalitat, continua abierta y judicializada.

${ }^{3}$ Si bien la CE consagra un Estado políticamente descentralizado, al mismo tiempo, instituye la provincia como una entidad local y como ámbito de prestación de servicios que no son trasferidos a los ejecutivos autonómicos. Esta situación ha permitido el mantenimiento de un aparato administrativo centralista (la administración periférica del Estado) liderado por el Subdelegado del Gobierno (antes gobernadores civiles) en cada provincia, a la que se añadió la figura del Delegado del Gobierno, representante del Ejecutivo central y de asumir la dirección de la administración periférica en cada comunidad autónoma. 
La creación de órganos e instrumentos ${ }^{4}$ para la articulación de la praxis competencial no ha cubierto las expectativas generadas puesto que su eficacia y rendimiento depende de la voluntad política para cooperar. Por otro lado, la excesiva rigidez del sistema de convenios interautonómicos los convierte en poco operativos. Si bien la cooperación horizontal se establece en la Constitución, lo cierto es que reviste un carácter difuso e impreciso ${ }^{5}$. La doctrina es casi unánime en reconocer que la regulación actual de la cooperación horizontal dificulta la utilización de los convenios y acuerdos como medio para impulsar y consolidar en España un Estado autonómico cooperativo (Tajadura Tejada, 2011:68).

La Conferencia de Presidentes de las Comunidades Autónomas ${ }^{6}$ presenta un carácter vertical y nace como un órgano de máximo nivel político entre el Estado y las comunidades autónomas. Su homóloga horizontal, la Conferencia de los Gobiernos de las $\mathrm{CCAA}^{7}$ ha dado sus primeros pasos en la institucionalización de encuentros de carácter político que no entrañen vinculación jurídica. Este foro permite el intercambio de información, promueve la colaboración multilateral y normaliza la necesaria cooperación interadministrativa del Estado descentralizado. Sin embargo, las relaciones de colaboración han tenido un desarrollo peculiar, con tres dinámicas distintas que plantean problemas diversos: un desarrollo notable de los instrumentos de colaboración vertical, una evidente precariedad de esos instrumentos horizontales entre las CCAA y la incógnita que supone la Conferencia de Presidentes en el conjunto de los instrumentos de cooperación existentes (García Morales, 2008).

La misma cooperación entre la comunidades autónomas está llamada a ser uno de los grandes ejes de su desarrollo y una prueba de su madurez organizativa (Tajadura Tejada, 2011:66).

${ }^{4}$ Siguiendo a Jiménez Asencio (2010) los principales órganos e instrumentos para la articulación del ejercicio competencial entre las administraciones son los siguientes: a) órganos de cooperación como: conferencias de presidentes, conferencias sectoriales y comisiones de carácter bilateral; b) Instrumentos de cooperación como: convenios y acuerdos de colaboración, planificación y programación conjunta y desarrollo de protocolos generales; c) órganos estatales de participación autonómica en ámbitos sectoriales que afecten a sus competencias; d) políticas normativas que afecten a sus competencias; e) participación de las CCAA en el ámbito internacional mediante la presencia de la acción exterior de las comunidades autónomas.

Vid. http://www.seap.minhap.gob.es/web/areas/politica_autonomica/coop_autonomica.html

5 Artículo 145. 2 de la CE.

${ }^{6}$ Desde su creación en 2004, la Conferencia de Presidentes de las Comunidades Autónomas ha celebrado cinco reuniones, siendo la última en octubre de 2012 sobre Estabilidad Fiscal, Situación Económica y laboral de España. http://www.seap.minhap.gob.es/es/areas/politica_autonomica/coop_autonomica/Confer_Presidentes.html

7 El $\overline{9}$ de julio de 2008 se celebró en Zaragoza un foro entre las comunidades autónomas para tratar diversos aspectos del desarrollo de sus respectivos Estatutos de autonomía. En el VIII Encuentro celebrado en el año 2011, en Santiago de Compostela, se acordó transformar el modelo y crear la Conferencia de los Gobiernos de las Comunidades Autónomas, con la finalidad de promover la cooperación y la colaboración política y administrativa entre las comunidades autónomas En 2011 en Santander se celebró la I Conferencia de Gobiernos de las CCAA. Para conocer con más detalle estos encuentros se puede consultar en el siguiente enlace: http://www.conferenciacomunidadesautonomas.org/ 


\subsection{La financiación autonómica}

El sistema de financiación ${ }^{8}$ es uno de los elementos fundamentales del mapa autonómico junto al reparto competencial. Su correcto diseño es imprescindible para el correcto funcionamiento del Estado autonómico y ha de garantizar dos principios constitucionales: la autonomía y la igualdad.

Sin embargo, el modelo de financiación no es una cuestión resuelta sino deudora de su proceso de configuración histórico que ha determinado sus carencias e inercias institucionales. En el diseño autonómico coexisten varios sistemas de financiación diferenciados. Uno de los sistemas es el conocido como régimen general y los otros corresponden al modelo de financiación de el País Vasco y la Comunidad Foral de Navarra, que disfrutan de regímenes fiscales especiales basados en el concierto o el convenio económico que les permite recaudar directamente los impuestos y negociar periódicamente con las autoridades centrales su aportación a la hacienda estatal. Como resultado de estas fórmulas fiscales, tanto el País Vasco como Navarra gozan de autonomía financiera y de una renta fiscal por habitante superior a la media nacional.

Los recursos financieros de las CC.AA. ${ }^{9}$ vienen establecidos constitucionalmente y se articula un modelo sostenido por las transferencias estatales, por la fiscalidad cedida y por la propia fiscalidad que junto a la emisión de deuda ${ }^{10}$ son las fuentes de financiación de las políticas públicas autonómicas. El actual sistema no garantiza el cumplimiento efectivo del principio constitucional de igualdad, restringe la autonomía regional en la vertiente de ingreso ${ }^{11}$ y no facilita una adecuada y transparente rendición de cuentas de las distintas administraciones ante sus ciudadanos (De la Fuente, 2012).

El informe de FUNCAS (2012) recoge algunas percepciones sobre el sistema autonómico. Los rasgos principales que destacan son: a) el sistema autonómico de finan-

${ }^{8}$ El modelo de financiación se estableció en la Ley Orgánica de Financiación de las Comunidades Autónomas (LOFCA) de 1980, y la Ley del Fondo de Compensación Interterritorial de 1984. Se ha visto modificado por la reformas de ambas leyes en diferentes años, y por la Ley General de Estabilidad Presupuestaria de 2001, reformada en 2006, 2007 y 2010, por la reforma de la Constitución por la Ley Orgánica de Estabilidad Presupuestaria y Sostenibilidad Financiera de las Administraciones Públicas, que introduce controles mas rigurosos y sanciones severas para todas las administraciones públicas. Para conocer la evolución legislativa y el marco legislativo actual de la financiación de las CCAA consultar:

$\mathrm{http}: / /$ www.congreso.es/consti/constitucion/indice/sinopsis/sinopsis.jsp?art=157\&tipo=2

9 Artículo 157 de la Constitución Española.

10 Actualmente, los límites al endeudamiento de las CCAA se establecen en la Ley Orgánica 2/2012 de 27 de abril de Estabilidad Presupuestaria y Sostenibilidad Financiera, que desarrolla el precepto constitucional. El plan de apoyo a pago a proveedores y el Fondo de Liquidez Autonómica son mecanismos de financiación para sanear la difícil situación financiera de algunas comunidades autónomas que no tienen posibilidad de acceder a los mercados crediticios en un contexto de crisis económica y elevado desempleo.

${ }^{11}$ Las comunidades autónomas tienen una limitada capacidad normativa en tributos cedidos, que es nula en tributación indirecta y un escaso recorrido recaudatorio de sus propios tributos. Las CCAA de régimen foral no tienen esta limitación. Por otro lado, hay que tener en cuenta la rigidez de los gastos autonómicos y su elevada sensibilidad social -sobre todo en sanidad, educación y servicios sociales- junto a la limitada capacidad normativa que frena la obtención de mayores recursos fiscales por parte de las CCAA (Fernández Llera y Morán Méndez, 2013). 
ciación no se entiende y los ciudadanos no conocen sus líneas fundamentales, b) los ciudadanos señalan que el sistema carece de transparencia, c) los medios de comunicación no informan de manera ordenada y clara, d) crece la conciencia de que la financiación autonómica es una pieza clave en el equilibrio del sistema financiero público en su conjunto, a través de déficits y superávits y de la emisión de deuda pública autonómica $\left.^{12} \mathrm{y}, \mathrm{e}\right)$ sensación dominante de que el sistema no es equitativo, favoreciendo claramente a unas comunidades autónomas (Cataluña, País Vasco y Madrid) (PérezDíaz, Mezo y Rodríguez, 2012: 110) y perjudicando a otras, calificadas como pobres.

En conclusión, junto a un deficiente diseño encontramos que la opacidad, una mal comprensión por la ciudadanía y mucha confusión sobre el sistema de financiación son algunos de los rasgos del marco financiero vigente. Lo que sí es muestra una clara dependencia de la correlación de fuerzas políticas que gobierna en cada momento, de la coyuntura económica y sujeta a numerosas modificaciones legislativas. Toda la discusión sobre la equidad, la corresponsabilidad fiscal y el reparto financiero permanece abierta y es un asunto pendiente para una futura modernización autonómica.

\section{Balance de la experiencia modernizadora de las administraciones públicas autonómicas: Una visión de conjunto}

Una de las ideas más aceptadas en el desarrollo autonómico es la falta de visión estratégica para introducir innovaciones en su modelo organizativo y funcional, de modo que el marco burocrático de la Administración General del Estado fue el referente principal en la acomodación y expansión de los aparatos políticos administrativos regionales (RodriguezArana, 1996; Canales, 1996; Morata, 1992, Baena, 1990, Ramió y Subirats, 1996). La prioridad se centraba en dar solución a los problemas e incertidumbres políticos y dejar en segundo plano las cuestiones relacionadas con la gestión pública y la eficacia administrativa. El resultado fue que la estabilización del ámbito político se produjo a expensas del ámbito administrativo que cristalizó a partir de las inercias originadas durante la fase del traspaso de competencias, estructuras y empleados públicos (Ramió y Salvador, 2004).

Las principales explicaciones que arrojan luz sobre la no innovación administrativa en las recién creadas CC.AA. se refieren a la ausencia de otros modelos válidos como referentes, a la urgencia política del momento y a la existencia de ciertas condiciones sobre el traspaso de competencias (Rodríguez-Arana, 1996; Canales Aliende, 1996).

Un tratamiento comprensivo del fenómeno modernizador requiere entender que las CC.AA., no son realidades aisladas sino que forman parte de un sistema político, que interaccionan con el resto de administraciones multinivel en una dinámica constante de

12 Los españoles hemos asistido a una crisis de deuda pública de gran envergadura, desde 2008 a 2013, que nos ha hecho conscientes de la responsabilidad de las comunidades autónomas en el déficit público y de la necesidad que contribuyan a reducirlo para beneficio de toda la colectividad. Por consiguiente, la financiación expresa uno de los problemas fundamentales para el sostenimiento del sistema político español y pone de manifiesto la necesaria conveniencia de revisar y reformar la fórmula de financiación autonómica. 
retroalimentación como parte de una misma colectividad política. Es, en definitiva, en esta red interactiva entre CC.AA y AGE donde vamos enmarcar el balance modernizador de las administraciones públicas autonómicas en su conjunto.

Si bien en una primera etapa la prioridad era la institucionalización autonómica, hipersimbólica, destinada a transmitir y asentar la idea corporativa con la implantación de nuevas estructuras administrativas (Canales, 1996), a continuación se privilegió un enfoque de modernización y reforma administrativa que operaba bajo los principios de la NGP, que promovía una visión postburocrática, centrada en los resultados y con una orientación al cliente ciudadano. En una fase ulterior, el centro de la experiencia modernizadora radicó en la e-Administración con las potenciales posibilidades que abrían las nuevas tecnologías de la información y de la comunicación en las administraciones públicas para, finalmente, desembocar en una fase de reformas estructurales como respuesta a la crisis económica y fiscal cuyas secuelas perdurarán durante algún tiempo. Las experiencias modernizadoras tienen diferentes fechas en cada comunidad autónoma puesto que, de la misma manera, unas asumieron antes las competencias que otras, de idéntica forma unas iniciaron antes las adaptaciones a la modernización administrativa. Por ello, aquí se destaca una visión de conjunto de las principales tendencias que han permeado las administraciones multinivel.

Seguidamente se expondrá una clasificación de los ciclos de modernización administrativa de las CC.AA. y de la AGE, que sin ánimo de exhaustividad, se ha elaborado a partir de variadas fuentes (Baena del Alcázar, 1990; Bañón i Martínez, 1993; Canales, 1996, 2002; Villoria, 1996; Ramió y Subirats, 1996; Rodríguez-Arana, 1996; Bañón y Carrillo, 1997; Olías de Lima, 2001, 2002; Ramió y Salvador, 2004; Prats, 2004, 2010; Arenilla, 2010, 2011; Aurioles Martín, 2013; Aja, García Roca y Montilla, 2015).

\subsection{Primer ciclo ${ }^{13}$ de modernización (1980-1986): Supervivencia y búsqueda de legitimidad}

En esta primera etapa modernizadora los esfuerzos políticos se dirigen a procurar la adaptación de las estructuras administrativas al nuevo régimen democrático y a proyectos como la descentralización política y la construcción del Estado de bienestar. La mayor parte de las CC.AA. se crean a principios de los años ochenta, difiriendo en cada una de ellas tanto su vía de acceso constitucional como el ritmo de las competencias asumidas. Los procesos de descentralización político territorial implican una redefinición del papel del Estado, una nueva reubicación organizativa con tres niveles territoriales diferenciados: el central, el autonómico y el local. Sin embargo, la inercia institucional que estos procesos lleva aparejada mantienen las viejas estructuras que se

${ }^{13}$ Según la RAE, el término ciclo tiene diferentes acepciones. Para nuestros objetivos, un ciclo modernizador representa una secuencia de etapas temporales por las que atraviesa una determinada serie de actividades o acontecimientos que tienen entre sí una vinculación temática y que en nuestro caso consisten en la modernización administrativa autonómica. 
acomodan a la nueva configuración autonómica. Los aspectos fundacionales y la consolidación de las competencias son las cuestiones nucleares que preocupan a las nuevas autoridades autonómicas. Las reformas administrativas durante los años ochenta y principios de los noventa fueron poco ambiciosas, solo con vocación de solucionar aspectos concretos, fragmentarios o puntuales del aparato administrativo autonómico sin entrar al fondo de su funcionamiento. En esta primera ola reformista, cobra un papel protagonista la función pública. Según Rodriguez-Arana (1996), la falta de legislación estatal básica postconstitucional en materia de función pública hasta 1984 provocó que una gran parte de las CC.AA. realizasen reclutamientos singulares junto al traspaso de funcionarios de la AGE y de las diputaciones provinciales.

La Ley 30/1984 de Medidas para la Reforma de la Función Pública ${ }^{14}$ alumbró la aprobación de leyes autonómicas e introdujo cierto orden en los recursos humanos, si bien no se modificó el diseño burocrático de función pública.

Sintéticamente, estamos ante una fase de búsqueda de legitimidad y de institucionalización de las CC.AA., de interiorización de la cultura administrativa estatal, que se transmitió a los recién creados aparatos administrativos autonómicos, y de aspiración a lograr el máximo techo competencial como materialización del principio de autogobierno. Finalmente, uno de los grandes elementos de esta etapa es el ingreso de España en la Comunidad Europa -actual Unión Europea (UE)- que, andando el tiempo, ha sido determinante en las trayectorias modernizadoras de nuestras administraciones públicas multinivel.

\subsection{Segundo ciclo de modernización (1987-1996): El auge de la NGP}

Una vez institucionalizadas, las CC.AA. se preocuparían de las disfunciones organizativas y de los procesos de modernización y eficiencia, bajo la inspiración de los principios de la Nueva Gestión Pública. Dicho enfoque supondría un giro hacia las famosas tres $e$ - eficiencia, eficacia y economía- y hacia una cultura orientada a satisfacer a los ciudadanos-clientes mediante unos servicios públicos de calidad. Modernizar la Administración pública se vincula con desburocratizarla, dotarla de más flexibilidad y agilidad, con mayor control de los costes y más receptiva al ciudadano. El paradigma postburocrático

14 Esta ley incorpora instrumentos de gestión para mejorar la eficacia y la transparencia en la ordenación de los empleos públicos. Una de sus novedades es la incorporación del puesto de trabajo como elemento fundamental para gestionar a los empleados públicos. Aunque se siguen manteniendo los cuerpos - que se organizan en cinco grupos A, B, C, D y E, diferenciados según el nivel de titulación exigida para el ingreso en cada cuerpo-, la función pública toma como base el puesto de trabajo, estructurada en treinta niveles jerárquicos. La ley 30/1984 consiguió reducir el número de cuerpos, aumentar la movilidad horizontal, introducir el puesto de trabajo como elementos de planificación y racionalización de las estructuras administrativas y cambiar el sistema retributivo vinculándolo al puesto desempeñado y a la productividad (Villoria y Del Pino, 1997:50).

Hasta a llegada de la democracia, los cuerpos superiores -tecnócratas- tenían predominio en el conjunto del Estado y de reparto de poder. El advenimiento del pluralismo político, con la emergencia de partidos políticos, sindicatos y grupos de la sociedad civil supuso una disminución del poder de la alta función pública. 
emerge como una superación del modelo weberiano de pensar y de actuar la gestión pública. La dirección de los asuntos públicos pasa a considerarse desde una perspectiva más eficientista orientada a resultados frente al enfoque garantista y jurídico propio del Derecho Público. La AGE pretendió, en un impulso reformador, establecer un conjunto de medidas a partir del documento Reflexiones para la modernización del Estado que fue elaborado por el Ministerio de Administraciones Públicas en 1990. Desde comienzos de la década de los noventa los ejecutivos autonómicos se enfrentan a problemas que requieren un cambio estructural para responder al contexto político. Así, el cumplimiento de los requisitos de Maastrichtt, la insatisfacción ciudadana con los servicios públicos, las experiencias de modernización que tenían lugar en países de nuestro entorno y la incorporación de las nuevas tecnologías de la información impulsó a la adopción de nuevos procedimientos y reformas administrativas. La NGP había irrumpido con fuerza en la mayoría de democracias occidentales gracias a la difusión de obras como La reinvención del Gobierno (Osborne y Gaebler, 1992,1994), Crear una Administración Pública que funcione mejor y cueste menos (Al Gore, 1993) y documentos de la O.C.D.E. La transformación de la gestión pública (1997).

Los gobiernos multinivel asumen la nueva filosofía de gestión bajo y adoptan planes de modernización administrativa donde el ciudadano es el consumidor de los servicios públicos. Pero, además, del éxito de la NGP, con sus nuevas metodologías, propuestas y orientaciones (Olías de Lima, 2001:12-20), este ciclo modernizador incorpora como novedades los Acuerdos Autonómicos ${ }^{15}$ (1992) que supone la finalización de la primera fase de grandes transferencias a las CCAA, tanto de la vía rápida como de la lenta de acceso y la entrada en vigor de la Ley 30/1992 $2^{16}$, de 26 de noviembre de Régimen Jurídico de las Administraciones Públicas y del Procedimiento Administrativo Común (LRJPAC).

En síntesis, esta etapa se caracteriza por una expansión autonómica, como expresión de autogobierno pero también de generación de una cultura institucional que hace suya la modernización administrativa y la europeización como elementos definidores propios.

\subsection{Tercer ciclo de modernización: La administración electrónica y la apuesta por la Calidad (1997-2004)}

En la primera década del siglo XXI, las dos grandes preocupaciones consisten en adaptar la administración a las nuevas tecnologías de la información y la comunicación, y profundizar en la obtención de unos servicios públicos de calidad. Los temas objeto de

15 Los Acuerdos Autonómicos de 1992 inician una uniformidad del mapa competencial que, a día de hoy, se cuestiona y que ha reactivado las controversias relacionadas con el hecho diferencial, como evidencia la cuestión catalana.

16 En esta ley se aborda la relación entre administraciones públicas desde el punto de vista del reparto competencial y desde el principio de cooperación. En su exposición de motivos se propone la simplificación de estructuras para todas las administraciones, el refuerzo de las garantías al ciudadano y reconoce que en una dinámica de modernización del Estado, el procedimiento administrativo es un instrumento para dinamizar el avance y una pieza básica en el proceso de modernización de la sociedad y de su administración. 
atención preferente pasan por reformas vinculadas a la informatización de los puestos de trabajo, la optimización de la gestión documental y procedimental, el rediseño de procesos, la reducción y racionalización de trámites burocráticos, la implementación de buenas prácticas organizativas y la implantación de la cultura de la calidad y de proximidad a la ciudadanía (Mendoza Mayordomo, 1990; Olías de Lima, 2001; Canales 2002; Arenilla 2012).

La pertenencia a la Unión Europea fomenta la implantación de la e-Administración, de la mano de Internet y la telefonía móvil. La UE se proponía promover el desarrollo y la difusión de las TICs entre todos los ciudadanos de los Estados miembros. Para ello se fijó un objetivo ${ }^{17}$ estratégico: convertirse en la economía basada en el conocimiento más competitiva y dinámica del mundo, capaz de crecer económicamente de manera sostenible con más y mejores empleos y con mayor cohesión social. La misma Comisión Europea, reconvertida en e-Comisión ${ }^{18}$ recuerda que las tecnologías de la Información y la Comunicación son la esencia misma de toda la administración moderna. En este marco estratégico, e-Comisión, ha liderado una administración electrónica de primer orden mediante la utilización de las TICs. En el desarrollo de este escenario digital, las administraciones españolas pusieron empeño de forma que la AGE promocionó la administración electrónica mediante las diferentes directivas europeas y la elaboración el Libro Blanco para la mejora de los servicios de 2000 y el Plan de Acción de la iniciativa Info XXI de 2001. Unos años después, aparecieron nuevas iniciativas que pretendían adaptarse a la Estrategia de Lisboa. ${ }^{19}$ El Libro Blanco

${ }^{17}$ La consecución de este objetivos requiere una estrategia global dirigida a preparar el paso a una economía y sociedad del conocimiento, basada en la sociedad de la información y de I+D, acelerando la competitividad y la innovación, con vistas para alcanzar el pleno empleo y reforzar la cohesión en la UE. Tales iniciativas se plasmarían en el Plan de Acción eEurope (Fuente: Consejo Europeo de Lisboa, 23 y 24 de marzo de 2000). http://www.europarl.europa.eu/summits/lis1_es.htm

${ }^{18}$ La e-Comisión o Comisión en línea; e-Commission 2006-2010: enabling efficiency and transparency. El propósito de la e-Comisión es aplicar a su propia administración una política de la sociedad de la información en el campo del e-gobierno, entendido como el empleo de la información y las nuevas tecnologías de comunicación en las administraciones públicas junto con el cambio de las organizaciones y la adquición de nuevas habilidades para mejorar los servicios públicos, los procesos democráticos y las políticas públicas. Se sirve de un modelo maduro de e-government basado en cuatro niveles de servicios electrónicos: a) nivel 1: simple site web, en el que la información se proporciona en línea sobre las políticas públicas y procedimientos administrativos, pero apenas registra cambios en la naturaleza de la interacción entre ciudadanos y administración; b) nivel 2: On-line government, que supone la articulación d mecanismos interactivos electrónicos simples como el correo electrónico para dar cuenta de mejores servicios a los ciudadanos; c) Nivel 3: Integrated government o gobierno integrado, que supone la automatización de la actividad administrativa procedente de una integración genuina y no una modificación simple de los procedimientos existentes para ser utilizados mediantes las TIC's. Sin embargo, algunos procedimientos administrativos utilizan el papel; d) Nivel 4: Transformed government o de gobierno transformado, que no requiere papel y la actuación administrativa está absolutamente automatizada en toda la organización y los servicios están dotados de más calidad y transparencia. http://ec.europa.eu/civil_service/admin/e_comm/index_es.htm;

$\mathrm{http} / / /$ ec.europa.eu/dgs/informatics/ecomm/doc/ecomm-2006-2010_cs_en_v414_postcis.pdf

19 La Estrategia de Lisboa tenía como objetivo estratégico para la UE convertirla en la economía más competitiva del mundo antes de 2010. La agenda Europa 2020 ha sustituido a la de Lisboa y pretende conseguir una economía comunitaria más inteligente, sostenible e integradora.

http://ec.europa.eu/europe2020/index_es.htm 
para la mejora de los servicios públicos de 2000 se planteó como una reflexión ante las exigencias de adaptación de e-Administración y del Plan de Calidad para la Administración General del Estado donde se enmarcan iniciativas como las cartas de servi$\operatorname{cios}^{20}$, la evaluación, la calidad en los servicios públicos, la formación de los empleados y directivos públicos, la receptividad, la flexibilización de las formas de gestión y la racionalización administrativa. La fuente teórica de estas metodologías hunde sus raíces en la NGP como el nuevo marco inspirador de la actuación pública.

En este ciclo modernizador, entra en vigor la Ley de Organización y Funcionamiento de la Administración General del Estado (LOFAGE) y la Ley del Gobierno, aprobadas en 1997, durante la VI legislatura. Por otro lado, se renuevan los proyectos de modernización autonómicos pero también se manifiestan pretensiones por parte de algunas CC.AA. de adaptar a la nueva realidad sus estatutos de autonomía para logar más capacidad de decisión política y el máximo nivel de competencias. En el año 2004 se crea la Conferencia de Presidentes de las Comunidades Autónomas y, en líneas generales, se inician procesos reivindicativos y se agravan las exigencias de diferenciación entre las comunidades autónomas, que habían estado larvados hasta entonces.

\subsection{Cuarto ciclo de la modernización (2005-2014). La década bifronte: de la e-Modernización a las reformas estructurales y la consolidación fiscal}

El último ciclo modernizador del que nos ocupamos plantea varias tendencias antagónicas, según se ubiquen antes o después del estallido de la crisis económica. En un primer momento se presentan tres tendencias como son la continuidad y el fortalecimiento de las iniciativas anteriores en materia de calidad y de administración electrónica; la introducción de instrumentos y mecanismos de gobernanza democrática y de buena administración y la aprobación de leyes destinadas a generar un cambio de la cultura burocrática (Arenilla, 2010). En una segunda fase, cuando estalla la virulenta crisis económica y fiscal, el término modernización administrativa dará paso al reformismo de las de reformas estructurales y las principales líneas de actuación se encaminarán hacia la consolidación fiscal ${ }^{21}$, la implementación de una batería de reformas estructurales fundamentadas en la necesidad de reducción del gasto público y de la deuda y la estimulación de la economía para detener la destrucción del empleo. De forma paralela, la existencia en la sociedad de una preocupación por la corrupción polí-

20 Se trata de un programa presentado al parlamento británico por el Primer Ministro, John Major, en 1991 cuyo principal objetivo era aumentar la calidad de los servicios públicos, difundiendo los compromisos de cobertura y las expectativas a satisfacer destinados a los usuarios. Los servicios públicos que los ciudadanos reciben debían satisfacerse mediante unos compromisos explícitos y públicos; además debían expresarse en términos de cobertura, costes o tiempos de espera. Los principios que enmarcaban las Cartas de Servicios pretendían dotar de transparencia el proceso porque los ciudadanos tenían derecho a saber, a obtener información con un lenguaje claro, completo y exacto, con posibilidad de elección, a la accesibilidad y a la no discriminación.

${ }^{21}$ La consolidación fiscal se sustenta en dos pilares básicos: la eliminación del déficit público estructural y la reducción de la deuda pública. 
tica administrativa y la degradación de la vida pública, en un contexto de insatisfacción y desconfianza hacia las instituciones representativas, conduce a la elaboración de medidas de transparencia y buen gobierno.

Este ciclo se presenta con dos caras, como Jano, y con resultados dependientes del rendimiento de la gobernanza económica y financiera multinivel, sobre todo en el ámbito comunitario. En una primera etapa, coincidiendo con la victoria electoral de los socialistas en 2004, en un contexto de bonanza y prosperidad, las tendencias del ciclo anterior se van consolidando. La implantación de las nuevas tecnologías crece exponencialmente, se generaliza la firma electrónica, los trámites electrónicos ${ }^{22}$, la remisión electrónica, la expansión de oficinas virtuales, hasta el punto que la terminología al uso se reconoce en expresiones como Sociedad de la Información, e-administración y e-democracia. Los planes Moderniza y Avanza pretendían introducir medidas de optimización, de eficiencia y flexibilidad al tiempo que profundizar la implantación de e-administración. El objetivo consiste en transitar de un modelo «Islas» a un modelo basado en la interoperativi$d a d^{23}$ y en la colaboración. El Plan Avanza y su continuación, el Plan Avanza 2 fueron las estrategias del gobierno en materia de Telecomunicaciones y Sociedad de la Información desde 2005 hasta la aprobación de la Agenda Digital en 2013.

Se aprueban diversos textos legales como la Ley 5/2006 de 10 de abril de Regulación de los conflictos de intereses entre miembros del Gobierno y altos cargos de la AGE, el Código de Buen Gobierno -cuya pretensión era servir de guía ética en la actuación de gobernantes y altos cargos-, la Ley 28/2006 de Agencias Estatales, para optimizar los servicios públicos, diseñar organizaciones más pequeñas, autónomas y flexibles en los servicios públicos y el Estatuto Básico del Empleado Público (EBEP) de 2007.

Por otro lado, los parlamentos regionales inician un período de actividad encaminada a la reforma de los Estatutos de Autonomía ${ }^{24}$. Las implicaciones de la oleada de reformas estatutarias suponen ampliación de las competencias para las comunidades autónomas. ¿Por qué precisamente en este ciclo se tensan las relaciones intergubernamentales? Para García Morales (2008:53), los nuevos estatutos de autonomía abrieron un debate sobre la bilateralidad y las vías específicas de colaboración entre el Estado

${ }^{22}$ Ley 11/2007 de acceso electrónico de los ciudadanos a los Servicios Públicos.

${ }^{23} \mathrm{La}$ interoperatividad se entiende como la capacidad mediante la cual sistemas heterogéneos, no solo tecnológicos, pueden intercambiar información y procesos técnicos o datos. Por tanto, la normalización técnica y el principio de cooperación administrativa conforman el sustrato de la interoperatividad para la mejora de las interacciones interadministrativas (Criado Grande, 2013:75).

${ }^{24}$ Reforma del Estatuto de Autonomía de la Comunidad Valenciana aprobado mediante la Ley Orgánica 1/2006 de 10 de abril; Reforma del Estatuto de Autonomía de Cataluña aprobado mediante la Ley Orgánica 6/2006 de 19 de julio; Reforma del Estatuto de Autonomía de Illes Balears aprobado mediante la Ley Orgánica 1/2007; de 28 de febrero; Reforma del Estatuto de Autonomía de Andalucía aprobado mediante la Ley Orgánica 2/2007 de 19 de marzo; Reforma del Estatuto de Autonomía de Aragón aprobado mediante la Ley Orgánica 5/2007 de 20 de abril; Reforma del Estatuto de Autonomía de Castilla y León aprobado mediante la Ley Orgánica 14/2007 de 30 de noviembre; Reforma de la Ley Orgánica de Reintegración y Amejoramiento del Régimen Foral de Navarra aprobada mediante la Ley Orgánica 7/2010 de 27 de octubre; Reforma del Estatuto de Autonomía de la Comunidad Autónoma de Extremadura aprobada mediante la Ley Orgánica 1/2011 de 28 de enero. (Fuente: Ministerio de Hacienda y Administraciones Públicas). http://bit.ly/1CvPHEB. 
y una comunidad determinada. Asimismo, a diferencia de otros países europeos, en España no existen plataformas de encuentro donde asistan solamente las CCAA., lo que dificulta la creación de una cultura de colaboración marcadamente horizonta ${ }^{25}$.

El estallido de la crisis económica en 2007 y la quiebra del sistema financiero global ${ }^{26}$ pusieron al mundo frente a una etapa de enorme inseguridad, desconfianza e incertidumbre. Con la crisis económica, financiera y de deuda a cuestas, las estrategias modernizadoras perdieron sustantividad y las medidas introducidas fueron por la línea de suprimir altos cargos y racionalizar ${ }^{27}$ estructuras administrativas. Desde el año 2010, la preocupación fundamental del Gobierno español ha sido cumplir el Pacto de Estabilidad y Crecimiento de la Unión Europea (PEC), que constituye el marco de gobernanza económica en el que se desenvuelve las administraciones públicas en pro de la eficiencia como objetivos fundamental. La modernización ha sido sustituida por el reformismo y la crisis ha relanzado el lenguaje de la economía pública: ajustes, consolidación fiscal, sostenibilidad, estabilidad presupuestaria, recortes ${ }^{28}$, control, deuda pública, racionalización del gasto público y disciplina fiscal. La gobernanza económica junto a una visión reformista y eficientista persiguen el objetivo final de la recuperación económica como justifica la Comisión para la Reforma de las Administraciones Públicas $\left(\mathrm{CORA}^{29}\right)$. La base teórica que fundamenta la Reforma de la Administración Pública es la economía pública privilegiando un enfoque tecnocrático-burocrático. Las reformas estructurales ${ }^{30}$ se consideran los pilares del desarrollo económico porque constituyen cambios que se introducen en las distintas esferas económica, fiscal, laboral, educativa y medioambiental para alcanzar los indicadores

25 Según García Morales (2008), en los últimos veinticinco años, el número de convenios horizontales suscritos entre las CCAA apenas llegan a treinta, mientras que los firmados con el Estado superan los 10.000.

${ }^{26}$ La ralentización de la economía de los Estados Unidos impidió a gran número de propietarios americanos reembolsar sus hipotecas -conocidas como subprime- y los bancos que tenían inversiones relacionadas con esas hipotecas empezaron a perder dinero. La declaración de quiebra de Lehman Brothers, cuarto banco de EEUU el 15 de septiembre de 2007, desencadenó la espiral perversa de la crisis globalizándola y deterioró las macromagnitudes económicas. Creció una alarma desconocida de forma que el temor y la desconfianza se extendieron y los bancos dejaron de prestarse dinero entre sí agravando la situación de los ciudadanos y empresas. Las economías europeas y sus sistemas financieros se vieron duramente afectados y los respectivos gobiernos nacionales tuvieron que intervenir con «rescates». En 2009, cuando Europa entró en recesión, el problema que en principio afectaba a los bancos, empezó a extenderse a los gobiernos, pues los mercados temían que algunos países no lograran rescatar a los bancos en dificultades. Los gobiernos se había acostumbrado a financiar sus presupuestos mediante grandes préstamos y habían contraído enormes deudas. Lo que comenzó como una crisis bancaria se convirtió en una crisis de la deuda soberana. A día de hoy, en marzo de 2015, las tasas de crecimiento de la eurozona siguen débiles, el consumo se ha contraído y los indicadores sociales muestran un crecimiento de las desigualdades que genera brechas sociales. http://bit.ly/1CvPMIy

${ }_{27}$ Real Decreto 494/2010, de 30 de abril, por el que se modifica el Real Decreto 1370/2008, de 1 de agosto, que desarrolla la estructura orgánica de la Presidencia del Gobierno.

${ }_{28}$ Por ejemplo, en los PGE se reducen los capítulos de gasto público; se suprimen organismos públicos dentro del Plan de Reestructuración y Racionalización del Sector Público Empresarial y Fundacional Estatal, congelación de la Oferta Pública de Empleo, Regulación de las retribuciones de empleados públicos (con supresión de la paga extraordinaria de diciembre de 2012), reducción de permisos y modificación de permisos, incompatibilidades e incapacidad temporal, etc.

${ }_{29}$ La Comisión para la Reforma de las Administraciones Públicas es un órgano adscrito al Ministerio de Hacienda y Administraciones Públicas, a través de la Secretaría de Estado de Administraciones Públicas. 
que tienen los países más desarrollados. Lo que se trata, finalmente, es de aumentar la productividad de la administración pública y a su vez, transmitirla a la economía nacional.

Seguidamente se presentan algunas de las medidas legislativas más significativas de esta segunda parte de la década bifronte en forma de tabla sintética, sin ánimo de exhaustividad. No pretende abarcar toda la producción legislativa ni siquiera sistematizar todas las variables o campos temáticos objeto de la atención reformadora del legislador así de los políticos. Para ello nos hemos detenido en la exposición de motivos de las leyes mencionadas donde se establecen las grandes líneas que se legislan y se han tomado las directrices principales.

Tabla 1: Síntesis de las principales variables acometidas por los paquetes legislativos y otras medidas reformistas (2010-2014)

\begin{tabular}{|c|c|c|c|c|c|c|c|c|c|c|c|}
\hline & 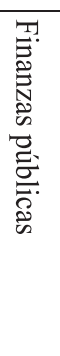 & 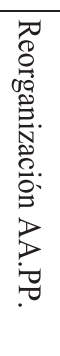 & 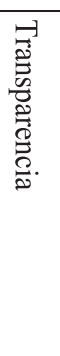 & 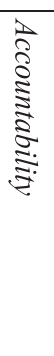 & 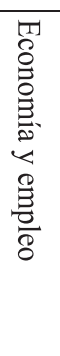 & 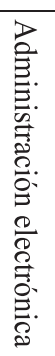 & 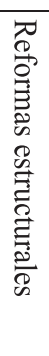 & 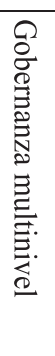 & 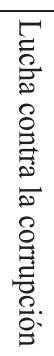 & 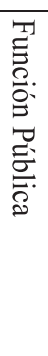 & 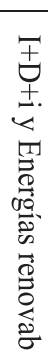 \\
\hline Ley Economía Sostenible 2011 & & & & & $\mathrm{X}$ & $\mathrm{X}$ & & & & & $\mathrm{X}$ \\
\hline $\begin{array}{l}\text { LO Estabilidad Presupuest. y } \\
\text { Sostenibilidad Financiera } 2012\end{array}$ & $\mathrm{X}$ & & $\mathrm{X}$ & $\mathrm{X}$ & $\mathrm{X}$ & $\mathrm{X}$ & $\mathrm{X}$ & $\mathrm{X}$ & & & \\
\hline CORA 2012 & $\mathrm{X}$ & $\mathrm{X}$ & $\mathrm{X}$ & $\mathrm{X}$ & $\mathrm{X}$ & $\mathrm{X}$ & $\mathrm{X}$ & $\mathrm{X}$ & & $\mathrm{X}$ & \\
\hline LRML (Mercado Laboral) & & & & & $\mathrm{X}$ & & $\mathrm{X}$ & & & & \\
\hline $\begin{array}{l}\text { Ley de Transparencia, acceso a } \\
\text { la información y Buen Gobierno } 2013\end{array}$ & & & $\mathrm{X}$ & $\mathrm{X}$ & & $\mathrm{X}$ & & & $\mathrm{X}$ & & \\
\hline $\begin{array}{l}\text { Ley de Racionalización y } \\
\text { Sostenibilidad Admon Local } 2013\end{array}$ & $\mathrm{X}$ & $\mathrm{X}$ & & $\mathrm{X}$ & & & $\mathrm{X}$ & $\mathrm{X}$ & & & \\
\hline Ley impulso factura electrónica... 2013 & $\mathrm{X}$ & $\mathrm{X}$ & $\mathrm{X}$ & $\mathrm{X}$ & $\mathrm{X}$ & $\mathrm{X}$ & $\mathrm{X}$ & & & & \\
\hline $\begin{array}{l}\text { Ley de Garantía unidad de } \\
\text { mercado } 2013\end{array}$ & & $\mathrm{X}$ & & & $\mathrm{X}$ & & $\mathrm{X}$ & $\mathrm{X}$ & & & \\
\hline $\begin{array}{l}\text { Ley de racionalización Sector } \\
\text { Público y medidas de reforma } \\
\text { adva. } 2014\end{array}$ & $\mathrm{X}$ & $\mathrm{X}$ & & & & $\mathrm{X}$ & $\mathrm{X}$ & & $\mathrm{X}$ & $\mathrm{X}$ & \\
\hline
\end{tabular}

Fuente: Elaboración propia.

${ }^{30}$ Entre las reformas estructurales de más calado destacamos: Ley 3/12 de Medidas Urgentes para la Reforma del Mercado Laboral, la Ley Orgánica de Estabilidad Presupuestaria, la Ley 19/2013 de Transparencia, acceso a la información y Buen Gobierno. La Ley 27/2013 de racionalización y sostenibilidad de la Administración Local, la Ley Orgánica 8/2013 para la Mejora de la Calidad Educativa y Ley 20/2013 de 9 de diciembre de Garantía de la unidad de Mercado. 


\subsubsection{CORA y la conflictividad autonómica: Una aproximación}

El informe CORA ${ }^{31}$, con diversidad de medidas de diferente enjundia, representa una iniciativa bajo la óptica del ajuste financiero para superar las ineficiencias administrativas-organizativas, según criterios tecnocráticos y económicos.

Con una clara orientación a la gobernanza económica -basada en la consolidación fiscal y en las reformas estructurales-, la reforma de las administraciones públicas sigue unas directrices principales basadas en la simplificación, la racionalización y la implantación de sistemas de medición de la eficiencia. La Ley 15/2014 de Racionalización del Sector público y otras medidas de reforma administrativa pone en marcha las propuestas de CORA sobre la reestructuración del sector público. La ley destaca que el objetivo de la reforma de las administraciones públicas no puede ser otro que convertir la Administración española en un factor de eficiencia y productividad, que posibilite el crecimiento económico y prestación efectiva de servicios públicos.

El informe Oficina para la Ejecución de la Reforma de Administración, conocida como OPERA ${ }^{32}$ analiza el grado de ejecución de las medidas, de las actuaciones propuestas y evalúa el grado de adhesión de las CC.AA. a la implantación de las medidas CORA. La finalidad es estudiar cada medida en cada comunidad autónoma a través de reuniones bilaterales entre OPERA y las Consejerías Autonómicas competentes. El Resumen Ejecutivo de la OCDE ${ }^{33}$ afirma que el mayor reto para el avance de CORA es el apoyo de las CC.AA., puesto que cualquier reforma sin el concurso autonómico estará abocada al fracaso. La OCDE alerta de la necesidad de un enfoque holístico y consensual en las reformas, buscando la coordinación e integración horizontal como una visión imprescindible en el proceso de diseño e implementación de las políticas, de modo que ayuden al gobierno a responder a la complejidad de los retos económicos y sociales ${ }^{34}$. Sin embargo, la conflictividad entre el Estado y las comunidades autónomas durante las tres últimas legislaturas y, sobre todo, desde el estallido de la crisis ha ido en aumento como se puede apreciar en el siguiente gráfico.

${ }^{31}$ La Comisión estableció cuatro subáreas temáticas para abordar sus propuestas: a) duplicidades administrativas, b) simplificación administrativa, c) gestión de servicios y medios comunes y, d) administración institucional. El informe CORA incluyó 217 propuestas, de las cuales 139 relacionan la Administración General del Estado (AGE) y las Comunidades Autónomas, y 78 solamente con la AGE.

32 Oficina para la ejecución de la Reforma de la Administración que presenta informes trimestrales y anuales al Consejo de Ministros sobre el progreso de la Comisión para la Reforma de las AAPP (CORA).

${ }^{33}$ Estudios de la OCDE sobre Gobernanza Pública. España: de la reforma administrativa a la mejora continua (2014).

${ }^{34}$ El colapso de las políticas públicas es una de las consecuencias de una política desbordada que incluye: un desbordamiento en cuanto escala, un desbordamiento respecto a discurso donde prima lo económico y un desbordamiento estructural que requiere asumir y gestionar la creciente complejidad de las políticas públicas (Brugué, 2014). 
Gráfico 1: Evolución de la conflictividad entre el Estado y las Comunidades Autónomas durante las tres últimas legislaturas (legislaturas VII 2004-2008, IX 2008-2011 y X 2011-2015)

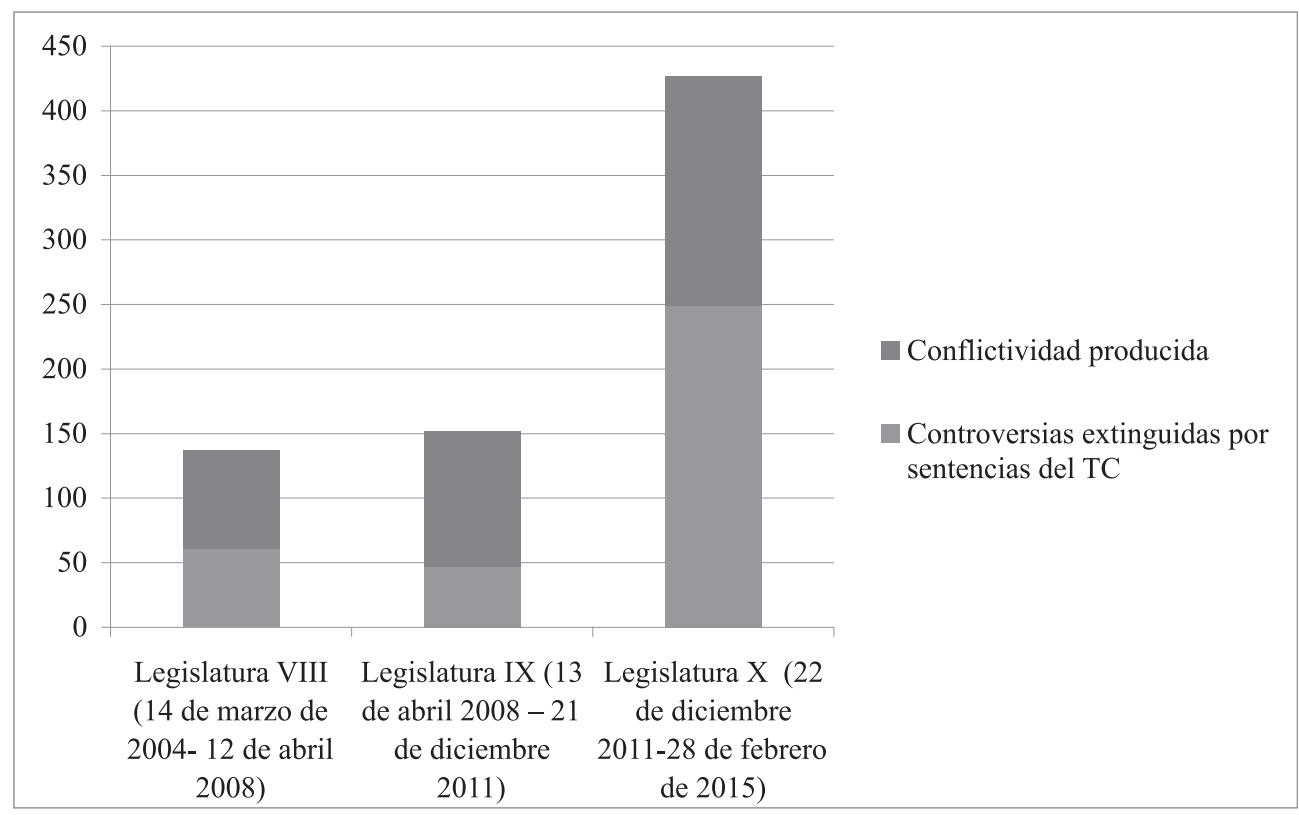

Fuente: Elaboración propia.

La escalada de acusaciones entre la AGE y las CCAA por la supuesta falta de responsabilidad, de lealtad institucional y de compromiso en los ajustes de las finanzas públicas resume uno de los discursos políticos más debatidos y polémicos del último lustro. La reconducción de tales disensos pasa, según la OCDE, por reducir los compartimentos estancos sectoriales y los déficits de coordinación y cooperación.

La OCDE afirma que la reforma de la administración pública es necesaria para minimizar el coste social de los ajustes y reconstruir la confianza en las instituciones y éste es el común desafío tanto de la AGE como de las comunidades autónomas.

\section{Conclusiones}

Tras esta visión panorámica del proceso de modernización del sistema político administrativo durante más de tres décadas, quedan muchos problemas pendientes de solución que serán materia de una futura modernización marcadamente autonómica. Entre los grandes retos se encuentran la necesidad de articular dispositivos de colaboración interadministrativos y de resolución de conflictos que no deriven en una judicialización permanente de los temas territoriales junto al establecimiento de un modelo de financiación que se corresponda con el modelo competencial vigente. Otro de los frentes conflictivos, 
y de cuyos efectos a largo plazo es pronto para evaluar desde la perspectiva autonómica, tiene que ver con la tutela presupuestaria y financiera del gobierno central sobre las haciendas territoriales y el modelo que resultará después de la crisis. Un estudio reciente ha analizado el impacto de las políticas de control del gasto público sobre los entes locales y sostiene que tales políticas de disciplina y control financiero han impuesto una mayor tutela financiera por la vía del control económico financiero. Este planteamiento resulta válido también para las CC.AA pues el escenario institucional resultante ha otorgado al Estado una mayor capacidad para ejercitar los controles sobre la actividad presupuestaria y las operaciones de endeudamiento (Aldeguer Cerdá, 2014: 214).

En resumidas cuentas, el éxito de los proyectos reformistas requiere la colaboración y la voluntad política de las comunidades autónomas en el marco de las relaciones intergubernamentales. Modernizar y reformar la administración pública ha de implicar una visión dinámica de las relaciones intergubernamentales como parte esencial de la gestión pública.

\section{Bibliografía}

Agranoff, R. (1997). «Las relaciones y la gestión intergubernamentales» en Bañón R. y Carrillo E. (coord.). La nueva administración pública. Alianza, Madrid.

Aguilar Villanueva, L. F. (2006). Gobernanza y gestión pública. FCE, México.

Aja, E., García Roca, J y Montilla, J. A. (2015). Informe comunidades autónomas 2014. Institut de Dret Public, Barcelona. En: http://idpbarcelona.blogspot.com.es/ (Consultado $7 / 03 / 2015)$.

Alda, M. y Ramos, J. A. (2010). «Cambio político y evolución de las relaciones intergubernamentales entre el Estado Central y las comunidades autónomas (1996-2008)», en Arenilla Sáez, M. (coord.). La administración pública entre dos siglos. Homenaje a Mariano Baena del Alcázar. Ed. Instituto Nacional de Administración Pública. Madrid, pp. 347-376.

Aldeguer Cerdá, B. (2014). «Autonomía local, equilibrio presupuestario y sostenibilidad financiera. Tendencias y perspectivas contemporáneas en las relaciones de tutela financiera y control presupuestario local en España (2008-2014)», en Cuadernos de Gobierno y Administración Pública, vol.1, $\mathrm{n}^{\circ}$ 2, pp. 181-218.

Aliende J.M. y Rodríguez Álvarez, J.M. (1997). La transformación de la gestión pública. La reforma en los países de la OCDE. Boletín Oficial del Estado, Madrid.

Arenilla Sáez, M. (2010). «Reformas organizativas: perspectivas histórica y comparada», en Sevilla, Jordi. (Dir.). La reforma de la Administración General del Estado, LID, Madrid, pp. 157-201.

Arenilla Sáez, M. (dir.). (2011). Crisis y reforma de la administración pública. Netbiblo, La Coruña.

Aurioles Martín, J. (2013). «El modelo autonómico: Desigualdades regionales, crisis económica y retos planteados», en eXtoikos, ${ }^{\circ} 10$, pp. 3-6.

Baena del Alcázar, M. (1990). Curso de ciencia de la administración. Vol. 1. Tecnos, Madrid. Ballart, X y Ramió, C. (2000). Ciencia de la administración. Tirant lo Blanch, Valencia.

Bañón i Martínez, R. (1993). «La modernización de la administración pública española. Balance y perspectivas», en Política y Sociedad, no 13, pp. 9-20.

Bañón i Martínez, R y Carrillo, E. (1997). La nueva administración pública. Alianza, Madrid. Barzelay, M. (2002). «La nueva gestión pública. Una invitación al diálogo globalizado. Estado, 
gobierno y gestión pública», en Revista Chilena de Administración Pública, nº 2, agosto, pp. 22-28.

Biglino Campos, P y Clara Mapelli, M. (dirs.). (2011). Garantías del pluralismo territorial. Fundación Manuel Giménez Abad, Zaragoza.

Brugué-Torruella, Q. (2014). «Políticas públicas: Entre la deliberación y el ejercicio de autoridad», en Revista Cuadernos de Gobierno y Administración Pública, vol. 1, no 1, pp. 37-55.

Canales Aliende, J. M. (1996). «Las administraciones autonómicas en España: ¿Imitación o innovación?», en Gestión y Análisis de Políticas Publicas, nº 7-8, pp. 139-42.

Canales Aliende, J. M. (2002). Lecciones de administración y de gestión pública. Universidad de Alicante, Alicante.

Canales Aliende, J. M y Pérez Guerrero, P. L. (2002). Introducción al gobierno y a la gestión local. Club Universitario, Alicante.

Canales Aliende, J. M y Menéndez Alzamora, M. (eds.) (2013). El sistema político administrativo valenciano. Tirant lo Blanc, Valencia.

Carro Fernández-Valmayor, J. L. (2011). Una introducción a la idea de modernización administrativa. Monográfico sobre Estudios sobre la modernización administrativa. Universidad de Santiago de Compostela, pp 5-20.

Conclusiones de la Presidencia del Consejo Europeo de Lisboa, 23 y 24 de marzo 2000. Consultado 7 de marzo de 2015. http://www.europarl.europa.eu/summits/lis1_es.htm

Criado Grande, J. I. (2013). «Interoperatividad y política sanitaria en España», en Barataria, ${ }^{\circ}$ 15 , pp. 73-94.

De la Fuente, A. (2012). «¿Qué reformas necesita el sistema de financiación regional?», en Papeles de Economía Española, $\mathrm{n}^{\circ} 133$.

E-Comision. (2006). Enabling efficiency and transparency. En: http://bit.ly/1CvLAIL (Consulta 7 de marzo de 2015).

Fernández Llera, R y Morón Méndez, E. (2013). «Las comunidades autónomas en la crisis: Cifras, planes y retos de control», en Auditoría y Gestión de Fondos Públicos, $\mathrm{n}^{\circ}$ 59, abril, pp. 39-50.

García Morales, M J J. (2002). «Relaciones de colaboración con las comunidades autónomas», en Informe Comunidades Autónomas 2001. En: http://www.idpbarcelona.net/cat/10iccaa/ 2001/1parte/ii/convenios.pdf

García Morales, M $\mathrm{M}^{\mathrm{a}}$ J. (2008). «Los instrumentos de las relaciones intergubernamentales», en Activitat Parlamentària, $\mathrm{n}^{\circ} .15$, pp. 48-62. En:

http://www.raco.cat/index.php/ActivitatParlamentaria/article/view/92006/117191

Gore, A. (1993). Creating a government that works better and costs less: Report of the national performance review. Random House, New York.

Jiménez Asencio, R. (2010). «La Administración General del Estado en un Estado compuesto» en Sevilla Jordi. (coord.). La reforma de la Administración General del Estado. LID Editorial Empresarial, Madrid.

López Nieto, L. (2006). «Los convenios de colaboración en el Estado Autonómico: intensidad, continuidad y multilateralidad de una relación casi desconocida», en López Nieto, L. (coord.). Relaciones intergubernamentales en la España democrática. Interdependencia, autonomía, conflicto y cooperación. Dykinson, Madrid, pp. 147-171.

Marando Vicent, L. y Patricia S. F. (1999). «La administración intergubernamental: El estado de la disciplina», en Naomí, B. y Aaron, W. (comp.). Administración pública. El estado actual de la disciplina. FCE, México.

Mendoza Mayordomo, X. (2000). «Técnicas gerenciales y modernización de la administración pública en España», en Documentación Administrativa, nº 223, julio, pp. 261-290. 
Ministerio de Administraciones Públicas. (1990). Reflexiones para la modernización del Estado. MAP, Madrid.

Ministerio de Administraciones Públicas. (2000). Libro Blanco para la mejora de los servicios públicos. MAP, Madrid.

Ministerio de Administraciones Públicas. (2005). Moderniza, Plan de Medidas 2006-2008 para la mejora de la Administración. MAP, Madrid.

Ministerio de Hacienda y Administraciones Públicas. (2012). Plan MEJORA 2012-2015. MHAP, Madrid.

Ministerio de Hacienda y Administraciones Públicas. (2013). Agenda Digital (sustituye al Plan Avanza y Plan Avanza 2). MHAP, Madrid.

Mondragón Ruiz de Lezana, J. (2001). «La gestión intergubernamental: Las relaciones intergubernamentales en España», en Olías de Lima Gete, B. (coord.). La nueva gestión pública. Pearson Educación, Prentice Hall, Madrid.

Morata, F. (1992). «Institucionalización y rendimiento político del Estado Autonómico. Un estudio comparado: Andalucía, Cataluña, Galicia y Comunidad Valenciana», en Revista de Estudios Políticos, vol. 76, pp. 255-297.

Morata, F. (2001). «El Estado de las Autonomías: Veinte años de rodaje» en Alcántara, M y Martínez, A. (eds.). Política y Gobierno en España. Tirant lo Blanc, Valencia.

Nieto, A. (1984). «Los estudios sobre la administración pública: La necesidad de construir una disciplina que sea la base de una clase directiva profesionalizada», en Documentación Administrativa, $\mathrm{n}^{\mathrm{o}}$ 200, enero-marzo, pp. 7-43.

Olías de Lima Gete. B. (Coord.) (2001). La nueva gestión pública. Pearson Educación, Prentice Hall, Madrid.

Olías de Lima Gete, B. (2002). «Las administraciones públicas españolas: Balance perspectivas», en Pastor G. y Crespo J. (coord.). Las administraciones públicas españolas. Balance y perspectivas. Graw Hill, Madrid.

Osborne, D y Gaebler, T. (1994). La reinvención del gobierno. Paidós, Barcelona.

Pérez-Díaz, V., Mezo, J y Rodríguez, J. C. (2012). «La crisis y las autonomías. La sociedad española ante la crisis económica y el sistema de autonomías». FUNCAS, Madrid.

Pérez Gabaldón, M. (2011). Los problemas competenciales en materia medioambiental y las relaciones intergubernamentales como posible vía de solución. Temas de las Cortes Valencianas, Valencia.

Pérez Gabaldón, M. (2013). La gestión intergubernamental de la política de cambio climático en España. Aranzadi, Navarra.

Prats, J. (2010). «Políticas de buena administración para la Administración General del Estado en España. Un enfoque de gobernanza democrática», en Ruiz-Huertas J. y Villoria M. (ed.). Gobernanza democrática y fiscalidad. Tecnos, Madrid.

Prats, J. (2004). «Las transformaciones de las administraciones públicas de nuestro tiempo», en Sainz Moreno, F. (dtor.). Estudios para la reforma de la Administración Pública. INAP, Madrid.

Ramió, C y Salvador, M. (2004). «El proceso de descentralización regional de España y el fenómeno de la no innovación ni mejora de las nuevas instituciones administrativas», en Studia Politicae, $\mathrm{n}^{\circ}$ 4, pp. 21-49.

Ramió, C y Subirats, J. (1996). «Los aparatos administrativos de las comunidades autónomas 1980-1995: Entre el mimetismo y la diferenciación», en Revista Vasca de Administración Pública, $\mathrm{n}^{\circ} 45$, pp. 151-181.

Ridaura Martínez, $\mathrm{M}^{\mathrm{a}}$ J. (2014). Representación política y parlamentarismo multinivel. Tirant Lo Blanch, Valencia. 
Ridaura, $\mathrm{M}^{\mathrm{a}} \mathrm{J}$. (2007). «Las comisiones bilaterales de cooperación en el sistema autonómico español», en Cuadernos constitucionales de la Cátedra Fadrique Furió Ceriol, n 60-61, pp. $65-84$.

Rodríguez-Arana Muñoz, J. (1996). «Reforma administrativa y Estado Autonómico», en Actualidad Administrativa, $\mathrm{n}^{\circ}$ 23, pp. 445-474.

Santolaya Machetti, P. (1984). Descentralización y cooperación. Instituto de la Administración Local, Madrid.

Villoria Mendieta, M. (1996). La modernización de la administración como instrumento al servicio de la democracia. INAP, Madrid.

Villoria Mendieta, M y Del Pino Matute, E. (1997). Manual de gestión de recursos humanos en las administraciones públicas. Tecnos, Madrid. 